\title{
Do We Still Need Phase 2 Trials? Late Obituary for Panitumumab and the SPECTRUM-Trial
}

Siano $M^{1 *}$ and Espeli $\mathbf{V}^{2}$

${ }^{1}$ Department of Internal Medicine, Clinic for Medical Oncology, Cantonal Hospital St. Gallen, Switzerland ${ }^{2}$ Department of Medical Oncology, Oncology Institute of Southern Switzerland, San Giovanni Hospital, Switzerland

*Corresponding author: Siano M, Department of Internal Medicine, Clinic for Medical Oncology, St. Gallen, Switzerland

Received: September 27, 2017; Accepted: October 18, 2017; Published: November 20, 2017

\section{Keywords}

Head and neck cancer; Panitumumab; Systemic treatment; Spectrum trial

\section{Editorial}

Head and neck squamous cell carcinoma (HNSCC) remains a disease with dismal prognosis in case of recurrence or in presence of metastases. Until the advent of anti-EGFR (epidermal growth factor receptor) antibodies $(\mathrm{AB})$ and in particular cetuximab, no treatment prolonging overall survival (OS) was established. With results from the EXTREME-Trial, comparing presumed standard platinumcontaining combination chemotherapy to the same regimen including cetuximab, a new standard in selected HNSCC patients with acceptable performance status became available [1].

A companion trial to EXTREME was also performed for panitumumab in HNSCC, called SPECTRUM-Trial [2]. Surprisingly, the OS benefit seen in EXTREME, was not reproducible. Better PFS, but only a trend towards a better OS, not being statistically significant, was shown. These results hampered the licensing of panitumumab for HNSCC by the manufacturer. This consequence was regretted by some head and neck oncologists, due to some favorable properties of panitumumab compared to cetuximab e.g. lower frequency and less severe allergic reactions, convenience in application.

Different, important reasons can be held accountable for the failure of the SPECTRUM-Trial and were extensively discussed in the literature. Besides the fact, that panitumumab shows less antibody-dependent cell-mediated cytotoxicity (ADCC) compared to cetuximab and this could contribute to better efficacy of cetuximab, there are some others. We want to mention only the most important ones: First of all, the lack of a maintenance treatment with the antiEGFR-AB after the initial chemotherapy combination part, which contributed in part to the good results from the EXTREME-Trial, because of some patients having long lasting responses, that are more and more recognized [3]. The comparator arm of SPECTRUM did extremely and unexpectedly well, even though the included patients had probably an unfavorable prognosis, compared to the ones included in EXTREME, due to primary tumor localization, performance status and some other reasons. But, it should be also remarked, that dosing of panitumumab in SPECTRUM was based on convenience and was adapted to concomitant chemotherapy administration and not based on soundly generated phase 2 data. Extrapolation from studies in other tumor entities, lead the investigators of SPECTRUM, to administer panitumumab every 21 days with a dose of $9 \mathrm{mg} / \mathrm{kg}$ body weight. It can be questioned, whether other tumor entities, with differing properties and expression patterns of cell surface receptors, respond the same way to targeting antibodies compared to HNSCC independently from dosing schedule.

One possible reason, was never properly considered, discussed or supported by clinical data so far, but risen by two phase 2 trials, recently published, investigating panitumumab in pre-treated HNSCC, and was the choice of the specific dosing schedule of panitumumab chosen for the SPECTRUM-Trial, which could have been sub-optimal. This choice, not based on phase 2 data, could confirm the notion, that moving directly from phase 1 to phase 3 trials, as it was the case for panitumumab in HNSCC, was, and probably is, not the best way to develop new anti-cancer treatments. The before mentioned phase 2 trials published recently, one by our group (PANI01-Trial) and the other by another group (PRISM-Trial), investigating panitumumab as mono therapy in pretreated, recurrent and metastatic HNSCC, seem to support this notion [4-6]. In these trials, different dosing schedules for panitumumab were chosen; $9 \mathrm{mg} / \mathrm{kg}$ per body weight every 21 days in PRISM and $6 \mathrm{mg} / \mathrm{kg}$ every 21 days in our trial. Even if no randomized comparison between these two dosing schedules was ever performed, from these trials a further explanation why SPECTRUM failed can be assumed. The 2-weekly schedule, applied in PANI01-Trial, could potentially be more efficacious and there is some evidence supporting this hypothesis.

In PANI01-Trial, patients with tumors harboring worse prognosis were included, with a lower number of oropharyngeal cancers and less HPV positive cases compared to patients included in the PRISM-Trial. Despite this fact, PFS of 2.6 months (95\% CI: 1.7 to 3.7 months) and OS of 9.7 months (95\% CI: 6.3 to 17.2 months) seem rather high, compared to the results published for the PRISM-Trial with a PFS of 1.4 months (95\% CI; 1.3 to 2.4 months)and an OS of 5.1 months (95\% CI; 4.3 to 8.3 months). Even if a direct comparison of these numbers is formally not permitted, drawing conclusions from these two trials, for a better choice for dosing schedule for further investigation in phase 3 trials, is admissible and standard practice. Even more, that differences in PFS and OS can be explained and could have been achieved due to the fact, that the two-weekly dosing schedule enabled the administration of a higher median adjusted drug exposure (42.9 mg/kg [range: 5.1-193.1 mg/kg] versus $26.8 \mathrm{mg} /$ $\mathrm{kg}$ [range, $8.2-198.2 \mathrm{mg} / \mathrm{kg}$ ]) than the one applied in PRISM. This is an important observation supporting our notion. Furthermore, since toxicity was comparable between both trials.
Ann Hematol Oncol - Volume 4 Issue 10 - 2017

ISSN : 2375-7965 | www.austinpublishing group.com

Siano et al. (@ All rights are reserved
Citation: Siano M and Espeli V. Do We Still Need Phase 2 Trials? Late Obituary for Panitumumab and the SPECTRUM-Trial. Ann Hematol Oncol. 2017: 4(10): 1177. 
If both trials results were available at the time of conception of the SPECTRUM-Trial, to our opinion, they could have led to a different, presumably more effective dosing schedule for panitumumab in HNSCC. The argument, that using panitumumab in combination with conventional chemotherapy (platinum and fluorouracil) is not the same setting as the one investigated in PANI01 and PRISM is valuable, but anyway, even the combination with chemotherapy should have been tested in a proper phase 2 study, before moving to phase 3. Moreover, if the fact is considered, that the primary end point of both trials (response rate) was not reached or satisfactory enough for further developing the drug in this setting. In absence of sound phase 2 data, with the combination of panitumumab and platinum/fluorouracil, it would not be justifiable these days, to plan and perform a phase 3 trial like SPECTRUM, and probably wasn't either at that time.

These statements are provocative, but confirm, the still valid notion, that phase 3 study design and dosing schedule should rely on proper phase 2 data and not only on assumptions and expert opinions. The strategy chosen by the investigators and the manufacturer, to rapidly move to phase 3 , hampered the potential of panitumumab in HNSCC, an antibody, that a lot of treating physicians would have liked to have in their armamentarium, showing good tolerability, and efficacy in terms of disease control.

We are convinced, that panitumumab has still a role as combination partner with novel immune therapies or targeted agents, and that efforts should still be undertaken, for the identification of predictive biomarkers for response. We therefore validated successfully a gene signature developed by a group in Milan, for prediction of long-lasting responses to cetuximab, on tissue available from the PANI01-Trial, trying to further understand which patients profit the most from anti-EGFR-AB treatment [3].

In conclusion, we think that phase 2 trials are still important for drug development and anti-EGFR-AB's should further be investigated in HNSCC.

\section{References}

1. Vermorken JB, Mesia R, Rivera F, Kawecki A, Rottey S, Erfan J, et al. Platinum-based chemotherapy plus cetuximab in head and neck cancer. $\mathrm{N}$ Engl J Med. 2008; 359: 1116-1127.

2. Vermorken JB, Stöhlmacher-Williams J, Davidenko I, Licitra L, Winquist E, Villanueva $\mathrm{C}$, et al. SPECTRUM investigators. Cisplatin and fluorouracil with or without panitumumab in patients with recurrent or metastatic squamouscell carcinoma of the head and neck (SPECTRUM): an open-label phase 3 randomised trial. Lancet Oncol. 2013; 14: 697-710.

3. Bossi P, Bergamini C, Siano M, Cossu Rocca M, Sponghini AP, Favales F et al. Functional genomics uncover the biology behind the responsiveness of head and neck squamous cell cancer patients to cetuximab. Clin Cancer Res. 2016; 22: 3961-3970.

4. Siano M, Molinari F, Martin V et al. Multicenter Phase II Study of Panitumumab in Platinum Pretreated, Advanced Head and Neck Squamous Cell Cancer. Oncologist. 2017;22(7):782-e70

5. Rischin D, Spigel DR, Adkins D, Wein R, Arnold S, Singhal N, et al. PRISM: Phase 2 trial with panitumumab monotherapy as second-line treatment in patients with recurrent or metastatic squamous cell carcinoma of the head and neck. Head Neck. 2015; 38: E1756-1761.

6. Siano M, Espeli V, Mach N. Gene expression prediction of panitumumab efficacy in head and neck squamous cancer. (unpublished data).
Ann Hematol Oncol - Volume 4 Issue 10 - 2017

ISSN : 2375-7965 | www.austinpublishing group.com

Siano et al. (C) All rights are reserved
Citation: Siano M and Espeli V. Do We Still Need Phase 2 Trials? Late Obituary for Panitumumab and the SPECTRUM-Trial. Ann Hematol Oncol. 2017; 4(10): 1177. 